6

\title{
THE USE OF A F. E. CODE FOR MULTICRITERIA RELIABILITY BASED OPTIMIZATION OF COMPOSITE MATERIAL STRUCTURES
}

\author{
A. Borri and E. Speranzini \\ Energetic Inst. - Structural Section, University of Perugia, \\ Str. S. Lucia Canetola, 06125 Perugia, Italy.
}

\begin{abstract}
A multicriteria reliability based optimization procedure for laminated composite material structures is presented. In order to solve the optimization problem, a multiobjective optimization criterion was applied which is capable of considering antagonist and non-comparable objective functions. By introducing parameters related to the reliability of the structures, the probability of failure with regard to a predefined limit state was considered among the objectives to optimize. The procedure has been implemented and coupled to a widely diffuse Finite Element code. This code is used both to solve the structural problem and to perform the entire reliability based optimization process by means of the minimization technique contained in the code itself.

As an example, a laminated composite shell in which the objective functions considered represent structural and economic performances is analyzed.
\end{abstract}

\section{RELIABILITY BASED OPTIMAL DESIGN}

In the case of laminated composite material structures, it is particularly interesting to perform an analysis that takes into account both structural optimization and structural reliability.

In these structures, the process of selecting the optimum solution is highly complex involving qualitative and quantitative factors at the same time. In fact, the structural optimization problem usually presents the difficulty of needing to consider objective functions which are non-comparable and cannot be combined to construct a single objective function. Furthermore, for laminated composite material 
structures the design variables are numerous so that multicriteria optimization may be an important tool for design.

On the other hand, some aspects of the problem regarding these structures are not deterministic, due also to the peculiar nature of the laminated material, and consequently the optimum design must deal with some uncertainties. In particular, considering random design parameters, it would be interesting to analyse the sensitivity of the optimal solutions with regard to random aspects.

If one introduces structural reliability as a further objective function (for example in terms of the probability of overcoming a predefined limit state), the problem can be posed as a "multicriteria reliability-based optimization" in which the objectives and all performance constraints are expressed as functions of the considered design variables.

In this paper, a procedure that considers the multicriteria optimization problem for composite material structures and the reliability at the same time is presented.

In order to solve the optimization problem, the multi-objective optimization criterion has been applied, which is capable of describing multi-objective function problems where the functions can be not only antagonist but also neither comparable nor commensurable.

As shown in previous papers [1-2], the multi-objective optimization criterion can be based on the concept of Pareto's optimum [3]. In this case, in which the objectives represent structural and economic performances that are non-comparable objective functions, the Pareto optimization problem was considered using the Trade-off method [4]. This method, through changing the vectorial problem into a scalar one, transforms the original problem into a series of simpler minimization problems with only one objective function that conveniently shuts out the others. The result of this optimization process is a set of "Pareto" optimal points, which are optimal in the sense that none of the objectives can be further improved without making at least one of the others worse. Therefore, infinite optimal solutions are possible among which the designer may choose the most applicable according to his necessities, and often on the basis of the indications given by the curve itself.

To introduce in the optimization process some aspects related to structural reliability, one or more objective functions that consider these aspects can be assumed.

In order to perform this process, a procedure has been implemented and added to a widely diffuse Finite Element code (ANSYS [5]) which is normally used in the deterministic field because the code in its standard form doesn't permit the use of random parameters.

In this work the optimization routine has been appropriately employed in order to optimize parameters related to structural reliability. To this aim, the procedure, having transformed the basic random variables into a space of standardized normally distributed variables, determines the minimum distance from the origin to the non-linear failure surface in the set of normalized variables using the minimization technique of the Finite Element code. In this way the code is used both to solve the structural problem and to perform the global optimization process. 


\section{THE PROCEDURE}

The proposed procedure is a point-by-point numerical process able to consider antagonist objective functions and random variables.

To determine the Pareto optimal solutions the methodology described in previous works [1-2] is used. This adopts the Trade-off Method that transforms the vector optimization problem into a scalar substitute one in which only one objective function $f_{i}$ (preference function or substitute objective function) is minimized while all the others $f_{i}(j \neq i)$ are kept bounded. By solving this minimization problem, a Pareto point is determined; by varying the values of the $f_{j}$ functions, other Pareto points can be calculated. Each point thus determined is Pareto optimum i.e. there is no other point in which the value of at least one objective function can be reduced without increasing the values of the others.

Since $P_{\text {fail }}$ (here defined as the probability of overcoming of a prefixed limit state) has been considered among the objective functions, two different problems have to be tackled:

- evaluating $P_{\text {fail }}$ when the value of each design parameter is fixed;

- individuating the values of the design parameters that minimize $P_{\text {fail }}$.

To solve the first problem, the minimum distance from the origin of the space of standardized independent normally distributed variables to a point on the failure surface is determined, having previously defined the failure condition. This minimization problem cannot be solved in closed-form because the analytical expression of the failure surface is not known (only the failure condition is known). However the minimization of the "distance" function between the origin and the points satisfying the failure condition can be numerically performed by the Ansys code. This distance permits to determine $P_{\text {fail }}=\Phi(-B)$. In any case, the value of this distance can be representative of $P_{\text {fail }}$ in the sense that a greater distance corresponds to a smaller $P_{\text {fail }}$ and viceversa.

For the second problem, it is sufficient to define the range of the design variables and use the Ansys optimization routine. This computes the point in the space of design variables to which corresponds the minimum value of $P_{f a i l}$ calculated as above described.

In the following, the algorithm is illustrated in the particular case of two objective functions (where $f_{1}$ represents $P_{\text {fail }}$ and $f_{2}$ is an antagonist function):

1. input parameters are defined;

2. a value for the objective function $f_{2}$ is fixed;

3. starting values for the design variables are chosen;

4. the transformation of the basic variables into uncorrelated and standardized normally distributed variables is performed;

5. the probability content in the failure set in standardized normally distributed variable space is evaluated: the optimization routine of the Finite Element code 
computes the minimum distance $B$ from the origin to a point on the failure surface as well as the corresponding $P_{\text {fail }}=\Phi(-B)$;

6. after having changed the values of the variables, steps 4 and 5 are repeated until the minimum value of $P_{f a i l}$ is obtained using the optimization routine of the code;

7. the point of $P_{f a i l}$ and $f_{2}$ co-ordinates represents a Pareto optimal point.

The external loop of the algorithm (from step 2 to step 7) is repeated to determine the other Pareto optimal points until the entire range of $f_{2}$ is covered; the internal loop (from step 4 to step 6 ) permits to minimize the function $f_{1}$ by varying the variable set.

The Pareto optimal set is therefore determined, the points of which are the optimal solution of the analysed problem.

The procedure is general and can also be applied in the case of basic variables that are not normally distributed and not mutually independent by performing the appropriate transformations.

\section{NUMERICAL EXAMPLE}

The procedure has been applied to analyse the laminated composite material structure of fig. 1 , representing a rectangular shell with a circular hole loaded by uniform pressure.

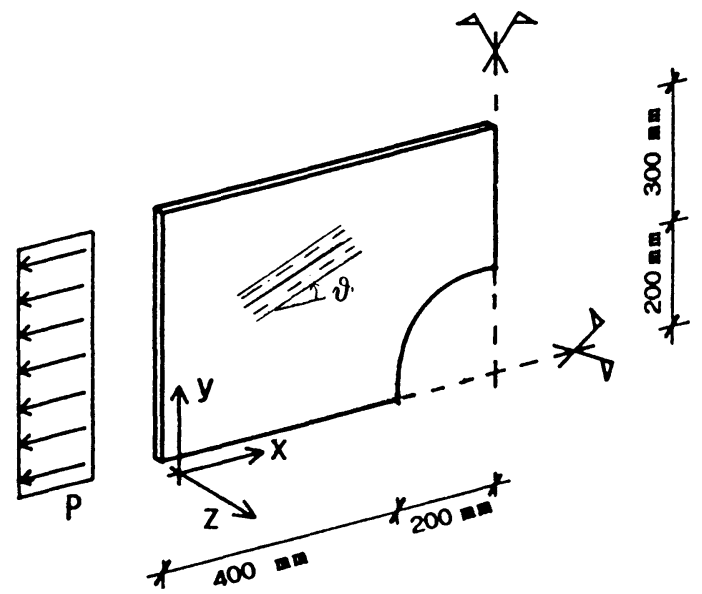

Fig. 1 - Shell composite structure ( load : $10^{3} \mathrm{~N} / \mathrm{mm}$ ) 
The material is a symmetrically cross-ply laminated composite that comprises of five orthotropic stacked layers having the sequence $\theta / 90^{\circ}+\theta / \theta / 90^{\circ}+\theta / \theta$; it consists of E-glass fiber and epoxy resin.

Regarding the composite material, the following basic assumptions have been made $[6,7]$ :

- the bonds between fibers and matrix are perfect;

- the fibers are regularly spaced and perfectly aligned with each other;

- the material of fiber and matrix is homogeneous, linearly elastic and isotropic;

- the constitutive laws of the fiber and the matrix considered are represented by the stress-strain curves of fig. 2.

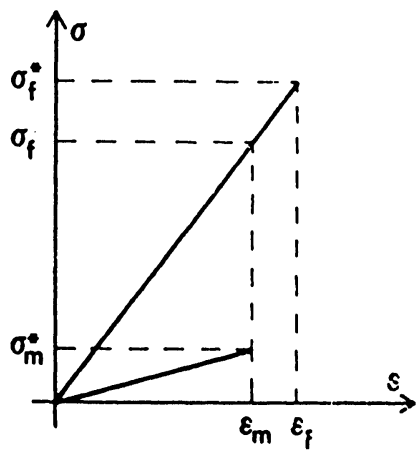

Fig. 2 - Constitutive laws for matrix ad fiber in the case of uniaxial tensile strength

The Pareto optimal solutions are determined by considering two objective functions: the probability of failure with regard to a limit state represented by the overcoming of the critical stress of the composite (using the maximum stress failure criteria), and the volume fraction of the fiber $V_{F}$ defined as the ratio between the volume of the fibers and the total volume of the composite. The problem is complex due to the characteristics of the material and to the fact that the functions $P_{f a i l}$ and $V_{f}$ (which represent the structural performance and the economic aspect respectively) are antagonist and cannot be combined in order to construct a single objective function.

Once $V_{f}$ is chosen as an objective function, the mechanical characteristics such as the elastic moduli, Poisson's coefficients and the critical stresses have to be determined at each loop of the procedure in terms of the properties of the fiber and the matrix and in terms of the relative fraction of their volume.

Being the considered composite material made up of E-glass fiber and epoxy resin and assuming $V_{F}$ in the range 0.2-0.6 (appropriate for most commercial laminates) the following failure strength in the composite (failure value with regard to evaluate $\left.P_{\text {fail }}\right)$ have been assumed [7-8]: 
$\sigma_{\mathrm{LT}}=\sigma_{\mathrm{f}}^{*} \mathrm{~V}_{\mathrm{f}} \quad$ longitudinal tensile strength

$\sigma_{\mathrm{TT}}=\sigma_{\mathrm{m}}^{*}\left(1-2\left(\mathrm{~V}_{\mathrm{f}} / \pi\right)^{1 / 2}\right) \quad$ transverse " "

$\sigma_{\mathrm{C}}=\mathrm{G}_{\mathrm{m}} /\left(1-\mathrm{V}_{\mathrm{f}}\right) \quad$ compression strength

where $\sigma^{*}$ and $\sigma^{*} \mathrm{~m}$ are the ultimate strength of fiber and matrix respectively and $\mathrm{G}_{\mathrm{m}}$ is the shear modulus of matrix. Failure for compressive strength in the fiber direction occurs when the fiber buckling for shear-mode is reached. At high $\mathrm{V}_{\mathrm{f}}$ $(0.2-0.6)$ the adjacent fibers buckle in the same wavelength and in phase with one another so that the matrix is subject to shearing deformation; this indicates that $\sigma_{\mathrm{c}}$ increases with $\mathrm{V}_{\mathrm{f}}$ but is controlled by the shear modulus of the matrix. Furthermore for this example it is reasonable to assume a transverse compressive strength comparable with the longitudinal compressive strength [6]. The stresses of eqs. (1) are the failure stresses as regards to which $P_{\text {fail }}$ is evaluated.

By taking into account the operative difficulties in the construction of the material, in this example, $V_{f}$ is considered to vary in a random fashion: consequently the composite strength (eqs. (1)) is also a random variable. Moreover, these aspects directly - and considerably at times - influence not only the critical stresses, but also the elastic moduli and Poisson's coefficients so that in the procedure they have to be adjusted in each step where the $V_{f}$ varies. $V_{f}$ is assumed to be normally distributed: the mean value is chosen in the range $0.2-0.6$ and the standard deviation is chosen as a fixed percentage of its mean value. So the mean value of the composite strength is the one which corresponds to the assumed value of $V_{f}$ and the standard deviation is the fixed percentage of this mean value. In order to minimize $P_{\text {fail }}$ the mean value of the normal distribution of the fiber direction $\theta$ is assumed to vary in the $0^{\circ}-45^{\circ}$ range while the standard deviation is fixed equal to $2^{\circ}$.

Each point of Pareto's curve has been calculated by fixing a value of $V_{f}$ and then computing the minimum of $\mathrm{P}_{\text {fail }}$ by means of the procedure presented. It can be noted that, once $V_{f}$ is fixed, $P_{\text {fail }}$ is a function of the random variable $\theta$. For each distribution of $\theta$ the procedure computes $P_{\text {fail }}$ and than chooses the minimum among the values determined.

Fig. 3 shows the resulting Pareto optimal curve $\left(P_{\text {fail }}\right.$ versus $\left.V_{f}\right)$ computed by considering standard deviation of $\mathrm{V}_{f}$ equal to a $20 \%$ of its mean value. The points of the curve are all equally optimal, i.e. optimal trade-off points between the structural and economical aspects. The shape of the curve indicates that some regions have greater interest than others, and gives useful quantitative information concerning the choice of a compromise solution. It can be seen, for example, that a choice of $V_{f}$ in the range $0.3-0.4$ can have a great practical interest because considerably small values of $\mathrm{P}_{\text {fail }}$ can be obtained with a relatively small value of $\mathrm{V}_{\mathrm{f}}$. 


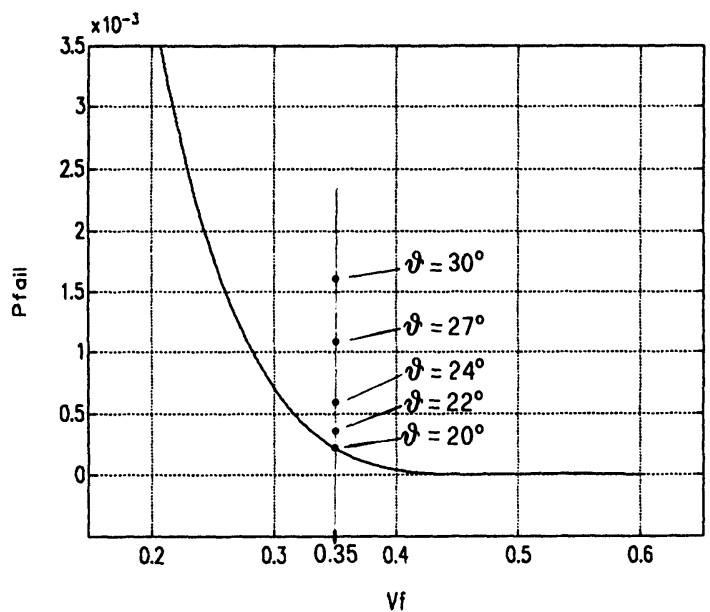

Fig. 3 - Pareto optimal curve

Furthermore, fig. 3 shows that a different value of $P_{\text {fail }}$ corresponds to each mean value of $\theta$. In the diagram are shown for example the different values assumed by $P_{\text {fail }}$ when $\theta$ varies, all corresponding to $V_{f}=0.35$. The point on the Pareto curve is that where the minimum values of $P_{f a i l}$ is obtained, and corresponds to $\theta=20^{\circ}$.

In fig. 4 Pareto curves corresponding to different values of the standard deviation of the $V_{f}$ probability distribution are plotted. It can be seen that the optimal value of the failure probability increases considerably when the standard

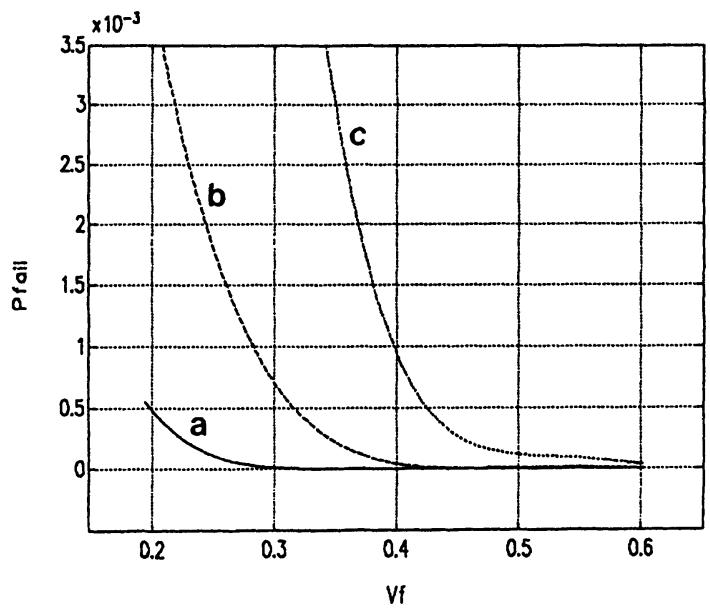

Fig. 4 - Pareto optimal curves (a,b,c curves correspond to $V_{f}$ standard deviation equal to $15 \%, 20 \%$ and $25 \%$ of its mean value, respectively) 
deviation of $V_{f}$ increases; furthermore, when $V_{f}$ assumes little values $P_{\text {fail }}$ is more affected by the dispersion of the values of the $V_{f}$ probability distribution.

\section{CONCLUSIONS}

A procedure that combines a structural optimization problem and a structural reliability one in the case of laminated composite material structures is presented. The procedure is formulated as a multicriteria optimization structural problem in which the probability of failure with respect to a predefined limit state is considered. To this aim, an algorithm that determines the Pareto optimal solutions with the probability of failure as an objective function has been performed. The procedure has been implemented and added to a widely diffuse finite element code considering uncorrelated normally distributed random variables.

Further researches will take into account not normally distributed and not mutually independent random variables and a new procedure for the inverse problem (multicriteria optimization with prefixed reliability) will be performed.

\section{REFERENCES}

1. Borri A., Speranzini E., Vetturini R., "Optimum Design of Laminated Composite Material Structures", CADCOMP 92, Delaware, USA, 1992.

2. Borri A., Speranzini E., "Multicriteria Optimization of Laminated Composite Material Structures", Meccanica, No 28, Kluwer Academic Publishers, Netherlands, 1993.

3. Koo D., "Elements of Optimizaton, with Application in Economic and Business", Heidelberg Science Library, 1977.

4. Eschenauer H.A., Kosky J., Osyczka A., "Multicriteria Design Optimization", Springer Verlag, Berlin, 1990.

5. ANSYS, "Seminar Notes: Design Optimization", Swanson Analysis System, Houston, Pa, USA, 1989.

6. Bhagwan D. A., Lawrence J. B., "Analysis and Performance of Fiber Composites", John Wiley \& Sons, New York, 1980.

7. Hull D., "An introduction to composite materials", Cambridge University Press, Cambridge, 1981.

8. Engineered Material Handbook, "Composites", Vol 1, ASM International, Metals Park, Hoio.

9. Augusti G., Baratta A., Casciati F., "Probabilistics Methods in Structural Engineering", Chapman and Hall Publishers, New York, 1984.

10. Casciati F., Faravelli L., "Fragility Analisys of Complex Structural Systems", John Wiley and Sons Inc., Tanton, England. 